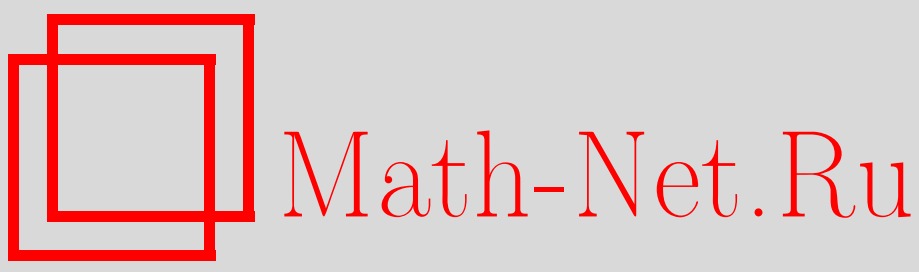

Е. Е. Тыртышников, Некоторые применения матричного признака равнораспределенности, Матем. сб., 2001, том 192, номер 12, 145-156

DOI: https://doi.org/10.4213/sm618

Использование Общероссийского математического портала Math-Net.Ru подразумевает, что вы прочитали и согласны с пользовательским соглашением

http://www . mathnet.ru/rus/agreement

Параметры загрузки:

IP: 3.80 .253 .173

26 апреля 2023 г., $10: 24: 56$ 
УДК $512.64+517.5$

\author{
Е.Е. Тыртышников
}

\title{
Некоторые применения матричного признака равнораспределенности
}

\begin{abstract}
Даны формулировки и простые доказательства теорем типа Сегё о распределении собственных и сингулярных чисел для матриц, полученных с помощью фиксированной конечной цепочки операций сложения, умножения и обращения из заданного конечного набора тёплицевых матриц с производящими функциями из $L_{1}$.

Библиографоия: 10 названий.
\end{abstract}

\section{§1. Введение}

В настоящей работе нас интересует следующий общий вопрос. Рассмотрим тёплицевы матрицы $A_{n}\left(f_{1}\right), \ldots, A_{n}\left(f_{m}\right)$ порядка $n$, порожденные функциями $f_{1}, \ldots$, $f_{m}$, и образуем новые матрицы $A_{n}$ с помощью фиксированной цепочки операций сложения, умножения и обращения, примененных к заданным матрицам и результатам предыдущих операций. Что можно сказать о распределении сингулярњых чисел (собственных значений) матриц $A_{n}$ ?

Этот вопрос изучался нами ранее в работе [1] в случае, когда $A_{n}$ есть произведение заданных или обратных к ним матриц и при этом все производящие функции принадлежат $L_{\infty}$. В качестве основного инструмента исследования использовались предложенный автором в [2], [3] матричный признак равнораспреденности и связь тёплицевых матриц с циркулянтными матрицами. В то время было не очень ясно, как переходить к рассмотрению производящих функций из $L_{1}$, поскольку прежняя связь с циркулянтными матрицами в этом случае казалась утраченной. В работе [4] удалось найти новую форму связи тёплицевых и циркулянтных матриц в случае $L_{1}$ при использовании фробениусовой матричной нормы (корень квадратный из суммы модулей элементов в квадрате).

Фробениусова норма привлекает простотой выражения через элементы матрицы. Заметим, однако, что матричньй признак равнораспределенности в изначальной форме [2] можно с легкостью применять и для других матричных норм, например для унитарно инвариантной 1-нормы Шаттена (сумма сингулярных чисел; см. [5]). Для данной нормы связь тёплицевых и циркулянтных матриц в случае $L_{1}$ не требует привлечения малоранговых матриц. Более того, в ряде случаев данная норма позволяет находить обобщения теоремы Сегё вообще без привлечения циркулянтных матриц (при внимательном чтении можно обнаружить применение

Работа вьполнена при поддержке Российского фонда фундаментальных исследований (грант № 99-01-00017).

(C) Е.Е. Тыртышников 2001 
этой нормы и в книге [6]). Безусловно, циркулянтные матрицы упрощают получение асимптотических распределений в простых случаях (для гладких производящих функций) и, вероятно, могут усложнять рассуждение в более сложных случаях (для производящих функций из $L_{1}$ ), когда, отказавшись от них, удается найти более прямой путь для обобщения.

В настоящей работе мы используем циркулянтные матрицы именно в простых случаях - когда они делают результат почти очевидным, а при переходе к производящим функциям из $L_{1}$ их использование уже оказьвается не столь обязательным. Что же касается малоранговых матриц в матричном признаке равнораспределенности, то их присутствие при изучении спектральных распределений в случае матричных операций с тёплицевыми матрицами, порожденными функциями из $L_{1}$, является существенньм.

\section{§2. Асимптотические распределения и равнораспределенность}

Фиксируем некоторое множество тестовых функций $\mathscr{F}$. Будем говорить, что последовательность кортежей $\left\{\lambda_{l n}\right\}_{l=1}^{n}$ имеет асимптотическое распределение $f(x),-\pi \leqslant x \leqslant \pi$, если для любой функции $F \in \mathscr{F}$ имеет место предельное соотношение

$$
\lim _{n \rightarrow \infty} \frac{1}{n} \sum_{l=1}^{n} F\left(\lambda_{l n}\right)=\frac{1}{2 \pi} \int_{-\pi}^{\pi} F(f(x)) d x
$$

Выбор отрезка $[-\pi, \pi]$ в качестве области определения $f(x)$ не является принципиальным. При определении класса гладкости для $f(x)$ полагаем, что $f(x)$ определена на всей вешественной оси и является $2 \pi$-периодической.

Две последовательности кортежей $\left\{\lambda_{l n}\right\}_{l=1}^{n}$ и $\left\{\mu_{l n}\right\}_{l=1}^{n}$ называются равнораспределенными, если для любой $F \in \mathscr{F}$ имеем

$$
\lim _{n \rightarrow \infty} \frac{1}{n} \sum_{l=1}^{n} F\left(\lambda_{l n}\right)=\lim _{n \rightarrow \infty} \frac{1}{n} \sum_{l=1}^{n} F\left(\mu_{l n}\right)
$$

С выбором множества тестовых функций связаны содержательность самих определений и удобство их применений. Следуя [2], [7], полагаем, что $\mathscr{F}$ состоит из всех вещественнозначных непрерывных функций $F(z)$ комплексной переменной $z$ с компактным носителем.

В дальнейшем будем предполагать, что все числа $\lambda_{\ln }$ вещественны и $f$ - измеримая функция.

Пусть символ \# $M$ обозначает число элементов в конечном множестве $M$. Для любой точки $z$ и $\delta>0$ рассмотрим величину

$$
\Gamma_{n}(\delta)=\#\left\{l:\left|\lambda_{l n}-z\right|<\delta, 1 \leqslant l \leqslant n\right\} .
$$

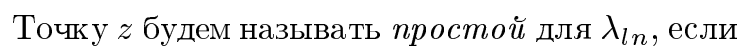

$$
\lim _{\delta \rightarrow 0} \limsup _{n \rightarrow \infty} \frac{\Gamma_{n}(\delta)}{n}=0 .
$$


Точку $z$ будем называть простой для $f(x)$, если

$$
\lim _{\delta \rightarrow 0} \mu\left(\mathscr{M}_{\delta}(z)\right)=0
$$

где $\mu\left(\mathscr{M}_{\delta}(z)\right)$ обозначает лебегову меру множества

$$
\mathscr{M}_{\delta}(z)=\{x:|f(x)-z|<\delta, \pi<x \leqslant \pi\}
$$

Любую точку, не являющуюся простой, будем называть точкой накопления. Легко видеть, что число точек накопления всегда не более чем счетно.

ЛЕмма 1. Последовательность кортежей $\left\{\lambda_{l n}\right\}_{l=1}^{n}$ имеет асимптотическое распределение $f(x)$ тогда и только тогда, когда соотночение (1) выполняется для характеристической функиии F любого отрезка, концы которого являются простыми точками для $f(x)$. При этом точки накопления для $\lambda_{\ln }$ и $f(x)$ одни и те же.

ЛЕмма 2. Последовательности $\left\{\lambda_{l n}\right\}_{l=1}^{n} u\left\{\mu_{l n}\right\}_{l=1}^{n}$ являются равнораспределенными тогда и только тогда, когда соотношение (2) выполняется для характеристической функции $F$ любого отрезка, конць которого являются простыци точками для $\lambda_{l n}$ и $\mu_{l n}$. При этом точки накопления для $\lambda_{l n}$ и $\mu_{l n}$ одни и те же.

Доказательства элементарны и поэтому опускаются.

Всюду далее предполагается, что $\lambda_{l n}$ и $\mu_{l n}$ суть сингулярные числа матриц $A_{n}$ и $B_{n}$ порядка $n$ соответственно. Основные утверждения сохраняют силу и в тех случаях, когда это собственные значения некоторых эрмитовых матриц $A_{n}$ и $B_{n}$. Сингулярные числа и собственные значения матрицы $A$ будем обозначать через $\sigma_{l}(A)$ и $\lambda_{l}(A)$ соответственно. Для определенности будем считать, что сингулярные числа (и собственные значения, если матрица $A$ эрмитова) упорядочены по невозрастанию. Введем следующие обозначения для компонент эрмитова разложения матрицы $A$ :

$$
H(A)=\frac{1}{2}\left(A+A^{*}\right), \quad K(A)=\frac{1}{2 \mathbf{i}}\left(A-A^{*}\right) .
$$

Теперь дадим обшую формулировку матричного признака равнораспределенности (в работах [1], [3], [4] приводилась и применялась его частная форма, связанная с фробениусовой нормой).

Функцию $G(x)$ будем называть $G$-функцией, если $G(0)=0$ и при $x \geqslant 0$ она является непрерьвной неотрицательной строго возрастающей функцией.

Лемма 3. Пусть задань две последовательности матрии $A_{n}$ и $B_{n}, u$ предположим, что существует $G$-функиия $G(x)$ и положительнье числа $c_{1}, c_{2}$ такие, что для любого $\varepsilon>0$ имеет место расщепление

$$
A_{n}-B_{n}=E_{n}+R_{n}
$$


$2 \partial e$

$$
\begin{gathered}
\sum_{l=1}^{n} G\left(\sigma_{l}\left(E_{n}\right)\right) \leqslant c_{1} \varepsilon n, \\
\quad \operatorname{rank} R_{n} \leqslant c_{2} \varepsilon n,
\end{gathered}
$$

для всех достаточно больиих $n \geqslant N(\varepsilon)$. Тогда сингулярные числа матрии $A_{n}$ и $B_{n}$ распределены одинаково. Равнораспределенными являются также собственные значения әрмитовых матрии $H\left(A_{n}\right)$ и $H\left(B_{n}\right)$ и собственные значения әрмитовых матрии $K\left(A_{n}\right)$ и $K\left(B_{n}\right)$.

Если $G(x)=x^{p}, p \geqslant 1$, то неравенство (6) имеет вид

$$
\left\|E_{n}\right\|_{(p)}^{p} \leqslant c_{1} \varepsilon n
$$

где $\|\cdot\|_{(p)}$ обозначает $p$-норму Шаттена, определяемую как корень $p$-й степени из суммы $p$-х степеней сингулярных чисел матрицы. В случае $p=2$ эта норма совпадает с фробениусовой нормой. При $p=\infty$ получаем хорошо известную спектральную норму, равную максимальному сингулярному числу.

Теперь сфформулируем еще один, более общий матричньй признак равнораспределенности.

Пусть для каждого $\varepsilon>0$ определена последовательность кортежей $\left\{\mu_{l n}(\varepsilon)\right\}_{l=1}^{n}$, и предположим, что для любой тестовой функции $F \in \mathscr{F}$

$$
\limsup _{n \rightarrow \infty}\left|\frac{1}{n} \sum_{l=1}^{n} F\left(\mu_{l n}(\varepsilon)\right)-\frac{1}{2 \pi} \int_{-\pi}^{\pi} F(f(x)) d x\right| \leqslant \delta_{F}(\varepsilon)
$$

где

$$
\lim _{\varepsilon \rightarrow 0} \delta_{F}(\varepsilon)=0
$$

В этом случае будем говорить, что числа $\mu_{l n}(\varepsilon)$ имеют предельное асимптотическое распределение $f(x)$ при $\varepsilon \rightarrow 0$, и будем писать

$$
\mu_{l n}(\varepsilon) \stackrel{\varepsilon \rightarrow 0}{\sim} f(x)
$$

Числа $\lambda_{l n}(\varepsilon)$ и $\mu_{l n}(\varepsilon)$ называются равнораспределенными в пределе при $\varepsilon \rightarrow 0$, если

$$
\limsup _{n \rightarrow \infty}\left|\frac{1}{n} \sum_{l=1}^{n} F\left(\lambda_{l n}(\varepsilon)\right)-\frac{1}{n} \sum_{l=1}^{n} F\left(\mu_{l n}(\varepsilon)\right)\right| \leqslant \delta_{F}(\varepsilon),
$$

где для $\delta_{F}(\varepsilon)$ имеет место соотношение $(9)$. В этом случае будем писать

$$
\lambda_{\ln }(\varepsilon) \stackrel{\varepsilon \rightarrow 0}{\sim} \mu_{\ln }(\varepsilon)
$$


ЛЕмма 4. Пусть $c_{1}, c_{2}$ - положсительные константы и $G(x)$ - некоторая G-функиия. Предположим, что для каждого $\varepsilon>0$ определены последовательности матрии, $A_{n \varepsilon} u B_{n \varepsilon}$ и существуют расщепления

$$
A_{n \varepsilon}-B_{n \varepsilon}=E_{n \varepsilon}+R_{n \varepsilon}
$$

$2 \partial e$

$$
\begin{gathered}
\sum_{l=1}^{n} G\left(\sigma_{l}\left(E_{n \varepsilon}\right)\right) \leqslant c_{1} \varepsilon n \\
\quad \operatorname{rank} R_{n \varepsilon} \leqslant c_{2} \varepsilon n
\end{gathered}
$$

для всех достаточно больших $n \geqslant N(\varepsilon)$. Тогда

$$
\begin{gathered}
\sigma_{l}\left(A_{n \varepsilon}\right) \stackrel{\varepsilon \rightarrow 0}{\sim} \sigma_{l}\left(B_{n \varepsilon}\right) \\
\lambda_{l}\left(H\left(A_{n \varepsilon}\right)\right) \stackrel{\varepsilon \rightarrow 0}{\sim} \lambda_{l}\left(H\left(B_{n \varepsilon}\right)\right), \quad \lambda_{l}\left(K\left(A_{n \varepsilon}\right)\right) \stackrel{\varepsilon \rightarrow 0}{\sim} \lambda_{l}\left(K\left(B_{n \varepsilon}\right)\right) .
\end{gathered}
$$

ДокАЗАтЕльство. Рассмотрим случай $E_{n \varepsilon}=0$. Пусть $\chi(x)$ - характеристическая функция некоторого отрезка П. Как показано в [2], если $\operatorname{rank}(A-B) \leqslant r$, то число сингулярных чисел матрицы $A$, принадлежащих П, может отличаться от аналогичного числа для $B$ максимум на $r$. Поэтому согласно (13)

$$
\left|\frac{1}{n} \sum_{l=1}^{n} \chi\left(\sigma_{l}\left(A_{n \varepsilon}\right)\right)-\frac{1}{n} \sum_{l=1}^{n} \chi\left(\sigma_{l}\left(B_{n \varepsilon}\right)\right)\right| \leqslant c_{2} \varepsilon .
$$

Пусть теперь $\nu>0$ и вьполняется неравенство

$$
\left|F(x)-F_{\nu}(x)\right| \leqslant \nu
$$

где $F_{\nu}(x)$ - конечная взвешенная сумма характеристических функций $\chi_{k}(x)$ для отрезков $\Pi_{k}$ :

$$
F_{\nu}(x)=\sum_{k=1}^{m} \alpha_{k} \chi_{k}(x), \quad m=m(\nu)
$$

Находим

$$
\lim _{\varepsilon \rightarrow 0} \limsup _{n \rightarrow \infty}\left|\frac{1}{n} \sum_{l=1}^{n} F\left(\sigma_{l}\left(A_{n \varepsilon}\right)\right)-\frac{1}{n} \sum_{l=1}^{n} F\left(\sigma_{l}\left(B_{n \varepsilon}\right)\right)\right| \leqslant 2 \nu .
$$

В силу произвольности $\nu$ левая часть неравенства есть нуль. Таким образом, случай $E_{n \varepsilon}$ разобран полностью.

Перейдем к общему случаю. В силу (12) неравенство

$$
\sigma_{l}\left(E_{n \varepsilon}\right) \leqslant G^{-1}(\sqrt{\varepsilon})
$$

имеет место для всех индексов $l=1, \ldots, n$, за исключением максимум $c_{2} \sqrt{\varepsilon} n$ из них. Поэтому можно найти матрицу $R_{n \varepsilon}^{\prime}$ такую, что

$$
\operatorname{rank} R_{n \varepsilon}^{\prime} \leqslant c_{1} \sqrt{\varepsilon} n
$$


и для матрицы $\widetilde{E}_{n \varepsilon} \equiv E_{n \varepsilon}-R_{n \varepsilon}^{\prime}$ спектральная норма (старшее сингулярное число) имеет оценку

$$
\left\|\widetilde{E}_{n \varepsilon}\right\|_{(\infty)} \leqslant G^{-1}(\sqrt{\varepsilon}) .
$$

Таким образом, справедливо расщепление

$$
A_{n \varepsilon}-B_{n \varepsilon}=\widetilde{E}_{n \varepsilon}+\widetilde{R}_{n \varepsilon}
$$

где $\widetilde{R}_{n \varepsilon}=R_{n \varepsilon}+R_{n \varepsilon}^{\prime}$ и, следовательно,

$$
\operatorname{rank} \widetilde{R}_{n \varepsilon} \leqslant\left(c_{1} \sqrt{\varepsilon}+c_{2} \varepsilon\right) n
$$

Для завершения доказательства достаточно рассмотреть случай $\widetilde{R}_{n \varepsilon}=0$. Возьмем произвольную тестовую функцию $F \in \mathscr{F}$ с модулем непрерывности

$$
\omega_{F}(\nu) \equiv \max _{\left|t_{1}-t_{2}\right| \leqslant \nu}\left|F\left(t_{1}\right)-F\left(t_{2}\right)\right|
$$

Поскольку для всех $l$

$$
\left|\sigma_{l}\left(A_{n \varepsilon}\right)-\sigma_{l}\left(B_{n \varepsilon}\right)\right| \leqslant\left\|A_{n \varepsilon}-B_{n \varepsilon}\right\|_{(\infty)},
$$

получаем

$$
\limsup _{n \rightarrow \infty}\left|\frac{1}{n} \sum_{l=1}^{n} F\left(\sigma_{l}\left(A_{n \varepsilon}\right)\right)-\frac{1}{n} \sum_{l=1}^{n} F\left(\sigma_{l}\left(B_{n \varepsilon}\right)\right)\right| \leqslant \omega_{F}\left(G^{-1}(\sqrt{\varepsilon})\right) .
$$

Остается заметить, что

$$
\omega_{F}\left(G^{-1}(\sqrt{\varepsilon})\right) \rightarrow 0 \text { при } \varepsilon \rightarrow 0
$$

Лемма доказана.

Отметим также следующее полезное утверждение.

Лемма 5. Пусть для каждого $\varepsilon>0$ определень матрицы $A_{n \varepsilon}$ и сингулярные числа $\sigma_{l}\left(A_{n \varepsilon}\right)$ имеют асимптотическое распределение $f_{\varepsilon}(x)$. Предполохим, что $f_{\varepsilon}(x)$ сходится по мере к измеримой функиии $f(x)$. Тогда $f(x)$ является предельным асимптотическим распределением для $\sigma_{l}\left(A_{n \varepsilon}\right)$ $n p u \varepsilon \rightarrow 0$. 


\section{§3. Тёплицевы и циркулянтные матрицы}

Рассмотрим формальный ряд Фурье

$$
f(x) \sim \sum_{k=-\infty}^{\infty} a_{k} e^{\mathbf{i} k x}
$$

и ассоциируем с ним тёплицевы матрицы

$$
A_{n}=\left[a_{k-l}\right], \quad 0 \leqslant k, l \leqslant n-1,
$$

а также два типа циркулянтных матриц: простые циркулянты $S_{n}$ и оптимальные циркулянты $C_{n}$. Напомним, что циркулянтная матрища $C=\left[c_{k l}\right]$ порядка $n$ определяется следующим свойством:

$$
c_{i j}=c_{k l} \quad \Leftrightarrow \quad i-j=k-l(\bmod n) .
$$

Таким образом, циркулянтная матрица определяется, например, элементами своего первого столбца. В простом ииркулянте первый столбец имеет элементы

$$
a_{0}, a_{1}, \ldots, a_{m}, a_{-m}, \ldots, a_{-1}
$$

если $n=2 m+1$, и

$$
a_{0}, a_{1}, \ldots, a_{m}, 0, a_{-m}, \ldots, a_{-1},
$$

если $n=2 m+2$. В оптимальном циркулянте первый столбец имеет элементы

$$
a_{0}, \frac{1}{n}\left((n-1) a_{1}+a_{-n+1}\right), \ldots, \frac{1}{n}\left(a_{n-1}+(n-1) a_{-1}\right) .
$$

Такой выбор обеспечивает минимум расстояния от $A_{n}$ до множества циркулянтных матриц, измеряемого по фробениусовой норме.

Пусть $f_{n}(x)$ обозначает $n$-ю частичную сумму ряда $(16)$, a $s_{n}(x)$ - соответствуюшую сумму Чезаро.

ЛЕмма 6 [2]. Для собственных значений простых и оптимальных ииркулянтов, ассочиированных с рядом (16), справедливы следующие формуль:

$$
\begin{array}{ll}
\lambda_{k}\left(S_{n}\right)=f_{[(n-1) / 2]}\left(\frac{2 \pi}{n} k\right), & 0 \leqslant k \leqslant n-1, \\
\lambda_{k}\left(C_{n}\right)=s_{n-1}\left(\frac{2 \pi}{n} k\right), & 0 \leqslant k \leqslant n-1 .
\end{array}
$$

Поскольку ряд Фурье сходится к $f(x)$ равномерно для любой $f \in C^{1}$, то из этой леммы следует, что сингулярные числа простых циркулянтов распределены как $|f(x)|$. Поскольку суммы Чезаро сходятся равномерно к $f(x)$ для любой $f \in C$, то заключаем, что сингулярные числа оптимальных циркулянтов распределены как $|f(x)|$ для $f \in C$. В этих же случаях вывод о распределении сингулярных чисел тёплицевых матриц $A_{n}$ можно сделать, используя матричньй признак равнораспределенности. 
Лемма 7 [2]. Ecлu $f \in L_{2}, m o$

$$
\left\|A_{n}-C_{n}\right\|_{F}^{2}=o(n) .
$$

То жее верно, если $C_{n}$ заменить на $S_{n}$.

Таким образом, мы с легкостью делаем вывод о том, что сингулярные числа тёплицевых матриц $A_{n}$ имеют асимптотическое распределение $|f(x)|$ в случае $f \in C$.

В работе [2] мы доказали, что сингулярные значения оптимальных циркулянтов имеют такое же распределение и в случае $f \in L_{2}$, а в силу (20) и леммы 3 это означает справедливость аналогичного утверждения и для $A_{n}$. Это предложение обобшает результат, полученный в работах [8], [9] при условии $f \in L_{\infty}$.

В случае вещественнозначной функции $f(x)$ матрицы $A_{n}, S_{n}, C_{n}$ являются эрмитовыми. Поэтому их собственные значения распределены как $f(x)$. Из вьшесказанного ясно, что этот факт имеет место для $f \in L_{2}$ и таким образом обобшает классическую теорему Сегё с условием $f \in L_{\infty}[6]$.

Если $f \in L_{1}$, то соотношение (20) теряет силу [4]. Чтобы получить обобщения теоремы Сегё на этот случай, в [4] было установлено существование матриц $R_{n}$ таких, что

$$
\left\|A_{n}-C_{n}+R_{n}\right\|_{F}^{2}=o(n), \quad \operatorname{rank} R_{n}=o(n) .
$$

Однако данный факт и сами обобщения можно получить и более простым способом. Вообще говоря, можно обойтись без оптимальных циркулянтов даже в случае $f \in C$, а вместо леммы 7 опираться на следуюшее легко проверяемое утверждение.

Лемма 8. Пусть ряд Фурье сходится $\kappa f(x)$ абсолютно. Тогда существуют положительные числа $c_{1}, c_{2}$ такие, что для любого $\varepsilon>0$ имеет место расщепление

$$
A_{n}-S_{n}=E_{n}+R_{n}
$$

əдe

$$
\left\|E_{n}\right\|_{(\infty)} \leqslant c_{1} \varepsilon, \quad \operatorname{rank} R_{n} \leqslant c_{2} \varepsilon n,
$$

для всех достаточно больших $n \geqslant N(\varepsilon)$. То же верно, если $S_{n}$ заменить на $C_{n}$.

ДокАЗАТЕЛЬСТво. В случае эрмитовых матриц данное предложение было доказано в [10]. В обшем случае рассмотрим эрмитовы разложения

$$
A_{n}=H\left(A_{n}\right)+\mathbf{i} K\left(A_{n}\right), \quad S_{n}=H\left(S_{n}\right)+\mathbf{i} K\left(S_{n}\right)
$$

и заметим, что $H\left(S_{n}\right)$ и $K\left(S_{n}\right)$ суть простые циркулянты, соответствующие тёплицевым матрицам $H\left(A_{n}\right)$ и $K\left(A_{n}\right)$. В то же время легко видеть, что $H\left(A_{n}\right)$ и $H\left(S_{n}\right)$ порождаются функцией $f^{H}(x)=(f(x)+\overline{f(x)}) / 2$, а $K\left(A_{n}\right)$ и $K\left(S_{n}\right)$ - функцией $f^{K}(x)=(f(x)-\overline{f(x)}) /(2 \mathbf{i})$. Для завершения доказательства заметим, что ряды Фурье для $f^{H}(x)$ и $f^{K}(x)$ сходятся абсолютно.

Таким образом, теорема Сегё и аналогичный результат для сингулярных чисел с очевидностью имеют место для абсолютно сходящихся рядов (16), в частности, когда $f \in C^{1}$. Чтобы обобщить эти результаты на случай $f \in L_{1}$, можно поступить следующим образом. 
Пусть значения функции $f(x)$ вешественны (почти всюду). Фиксируем произвольное $\varepsilon>0$ и рассмотрим ее $\varepsilon$-приближение $f_{\varepsilon}(x) \in C^{1}$ в смысле $L_{1}$. Положим

$$
g(x) \equiv f(x)-f_{\varepsilon}=g^{+}(x)-g^{-}(x),
$$

где $g^{+}(x)$ и $g^{-}(x)$ неотрицательны (почти всюду). Тогда

$$
\|g\|_{L_{1}}=\left\|g^{+}\right\|_{L_{1}}+\left\|g^{-}\right\|_{L_{1}} \leqslant \varepsilon
$$

Далее, $A_{n}\left(g^{+}\right), A_{n}\left(g^{-}\right)$- эрмитовы неотрицательно определенные матрицы и поэтому

$$
\begin{aligned}
\left\|A_{n}\left(g^{+}\right)\right\|_{(1)} & =\frac{n}{2 \pi}\left\|g^{+}\right\|_{L_{1}}, \\
\left\|A_{n}\left(g^{-}\right)\right\|_{(1)} & =\frac{n}{2 \pi}\left\|g^{-}\right\|_{L_{1}}
\end{aligned}
$$

Следовательно,

$$
\left\|A_{n}(g)\right\|_{(1)} \leqslant\left\|A_{n}\left(g^{+}\right)\right\|_{(1)}+\left\|A_{n}\left(g^{-}\right)\right\|_{(1)} \leqslant \frac{n}{2 \pi} \varepsilon .
$$

Таким образом, тёплицевы матрицы $A_{n}(f)$ и $A_{n}\left(f_{\varepsilon}\right)$ удовлетворяют условиям лемм 4 и 5. Отсюда вытекает, что $f(x)$ является предельным асимптотическим распределением для $\sigma_{l}\left(A_{n}\left(f_{\varepsilon}\right)\right)$ и одновременно асимптотическим распределением для сингулярных чисел матриц $A_{n}(f)$.

Случай комплекснозначной производящей функции $f(x)$ легко сводится к рассмотренному с помощью эрмитова разложения.

При тех же условиях нетрудно показать, что

$$
\left\|C_{n}(g)\right\|_{(1)} \leqslant\left\|C_{n}\left(g^{+}\right)\right\|_{(1)}+\left\|C_{n}\left(g^{-}\right)\right\|_{(1)} \leqslant \frac{n}{2 \pi} \varepsilon .
$$

Отсюда можно получить следуюшую форму связи тёплицевых и оптимальных циркулянтных матриц в случае $f \in L_{1}$.

Лемма 9. Eсли $f \in L_{1}$, mo

$$
\left\|A_{n}(f)-C_{n}(f)\right\|_{(1)}=o(n) .
$$

\section{§4. Распределения при матричных операциях}

Пусть $f_{1}, f_{2}, \ldots, f_{m} \in L_{1}$ и $A_{n}\left(f_{1}\right), A_{n}\left(f_{2}\right), \ldots, A_{n}\left(f_{m}\right)$ - соответствуюшие тёплицевы матрицы. Положим

$$
T_{0}=I, T_{1}=A_{n}\left(f_{1}\right), \ldots, T_{m}=A_{n}\left(f_{m}\right)
$$

(I - единичная матрица) и рассмотрим следующие бинарные операции:

$$
\begin{array}{ll}
\xi_{1}(A, B)=A B, & \xi_{2}(A, B)=A B^{-1}, \\
\xi_{3}(A, B)=A+B, & \xi_{4}(A, B)=A-B .
\end{array}
$$


При обращении матриц соответствующие матрицы предполагаются невырожденньми. Предположим, что $A_{n} \equiv T_{m+p}$ есть результат следующей цепочки $\mathscr{A}$ матричных операций:

$$
\begin{aligned}
& T_{m+1}=\xi_{k(m+1)}\left(T_{i(m+1)}, T_{j(m+1)}\right), \\
& \ldots \ldots \ldots \ldots \ldots \ldots \ldots \ldots \ldots \ldots \ldots \ldots \ldots \ldots \\
& T_{m+p}=\xi_{k(m+p)}\left(T_{i(m+p)}, T_{j(m+p)}\right),
\end{aligned}
$$

где для всех $1 \leqslant l \leqslant p$

$$
0 \leqslant i(m+l), j(m+l)<m+l, \quad k(m+l) \in\{1,2,3,4\} .
$$

Мы знаем, что сингулярные числа матриц $A_{n}\left(f_{l}\right)$ распределены как $f_{l}(x)$. Что можно сказать о сингулярных числах матриц $A_{n}=T_{m+p}$ ?

Для ответа на этот вопрос введем следующую цепочку соответствующих арифметических операций с производящими функциями:

$$
\begin{aligned}
& f_{m+1}=\xi_{k(m+1)}\left(f_{i(m+1)}, f_{j(m+1)}\right) \\
& \ldots \ldots \ldots \ldots \ldots \ldots \ldots \ldots \ldots \ldots \ldots \ldots \ldots \ldots \ldots \ldots \ldots \\
& f_{m+p}=\xi_{k(m+p)}\left(f_{i(m+p)}, f_{j(m+p)}\right) .
\end{aligned}
$$

Мы хотим показать, что при разумных общих предположениях (в частности, что деление имеет смысл) сингулярные числа матриц $A_{n}$ имеют асимптотическое распределение $f(x) \equiv f_{m+p}(x)$.

Лемма 10. Пусть $f \in L_{1} u \varepsilon>0$. Тогда существуют матрицы $A_{n \varepsilon} u$ ииркулянтные матриць $C_{n \varepsilon}$ такие, что

$$
A_{n}(f)=A_{n \varepsilon}+R_{n \varepsilon}^{\prime}, \quad C_{n}(f)=C_{n \varepsilon}+R_{n \varepsilon}^{\prime \prime}
$$

и при этом для всех достаточно больиих $n$

$$
\begin{gathered}
\left\|A_{n \varepsilon}\right\|_{(\infty)},\left\|C_{n \varepsilon}\right\|_{(\infty)} \leqslant M(\varepsilon) \\
\operatorname{rank} R_{n \varepsilon}^{\prime}, \operatorname{rank} R_{n \varepsilon}^{\prime \prime} \leqslant c \varepsilon n
\end{gathered}
$$

әде с не зависит от $\varepsilon$ и $n$.

ДокАЗАТЕльство. Сингулярные числа матриц $A_{n}(f)$ и $C_{n}(f)$ распределены как $|f(x)|$. Поскольку мера множества $\{x:|f(x)|>M\}$ стремится к нулю при $M \rightarrow \infty$, число сингулярных чисел матриц $A_{n}(f)$ и $C_{n}(f)$, превосходяших $M$, имеет вид $o(n)$. Матрицы $A_{n \varepsilon}$ и $C_{n \varepsilon}$ можно получить из сингулярных разложений $A_{n}(f)$ и $C_{n}(f)$ посредством модификации старших сингулярных чисел.

Лемма 11. Пусть $f \in L_{1}$ и матриць $A_{n}(f)\left(u C_{n}(f)\right)$ невырожсденные. Предположим также, что почти всюду $f(x) \neq 0$. Тогда сингулярные числа матрич, $A_{n}^{-1}(f)\left(\right.$ и $\left.C_{n}^{-1}(f)\right)$ имеют асимптотическое распределение $1 /|f(x)|$.

Если $|f(x)| \geqslant c_{0}>0$ почти всюду, то

$$
\left\|A_{n}^{-1}(f)\right\|_{(\infty)},\left\|C_{n}^{-1}(f)\right\|_{(\infty)} \leqslant c_{0}^{-1} .
$$


ДокаЗАтЕЛЬСтво. Рассмотрим отрезок $[a, b]$, концы которого не являются точками накопления для $f(x)$. Очевидно,

$$
\#\left\{i: a \leqslant \sigma_{i}\left(A_{n}\right) \leqslant b\right\}=\#\left\{i: b^{-1} \leqslant \sigma_{i}\left(A_{n}^{-1}\right) \leqslant a^{-1}\right\},
$$

и доказательство завершается использованием леммы 1.

Собственные значения циркулянтной матрицы $C$ с первым столбцом $c=\left[c_{0}, c_{1}\right.$, $\left.\ldots, c_{n-1}\right]^{T}$ имеют вид

$$
\lambda_{k}=\sum_{l=0}^{n-1} c_{k} e^{\mathbf{i} \frac{2 \pi}{n} k l}, \quad k=0,1, \ldots, n-1 .
$$

Будем говорить, что $f(x)$ является спектральной функцией для $C$, если

$$
\lambda_{k}=f\left(\frac{2 \pi}{n} k\right), \quad k=0,1, \ldots, n-1
$$

ЛЕмма 12. Предположим, что сингулярные числа матрии $A_{1 n}$ и $A_{2 n}$ имеют асимптотические распределения $f_{1}(x)$ и $f_{2}(x)$ соответственно, где $f_{1}(x)$

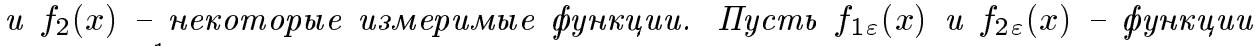
класса $C^{1}$, сходящиеся по мере $\kappa f_{1}(x)$ и $f_{2}(x)$ при $\varepsilon \rightarrow 0, u C_{1 n \varepsilon}, C_{2 n \varepsilon}-$ ииркулянтные матрицы со спектральными функциями $f_{1 \varepsilon}(x)$ и $f_{2 \varepsilon}(x)$ соответственно такие, что каждая из пар $A_{1 n}, C_{1 n \varepsilon}$ и $A_{2 n}, C_{2 n \varepsilon}$ удовлетворяет условиям леммы 4. Тогда сингулярные числа матрии $A_{n}=A_{1 n} A_{2 n}$ имеют асимптотическое распределение $|f(x)|$, где $f(x)=f_{1}(x) f_{2}(x)$, а сингулярные числа матрии $A_{1 n} \pm A_{2 n}$ распределень как $\left|f_{1}(x) \pm f_{2}(x)\right|$.

Доказательство сводится к проверке условий матричного признака равнораспределенности (лемма 4) для матриц $A_{n}$ и циркулянтных матриц $C_{n \varepsilon} \equiv C_{1 n \varepsilon} C_{2 n \varepsilon}$. Мы опираемся на то, что рассматриваемые матричные операции, применяемые к циркулянтньм матрицам, имеют своим результатом также циркулянтную матрицу; все циркулянтные матрицы одного порядка имеют общий базис собственных векторов, и собственные значения итоговой циркулянтной матрицы получаются с помощью той же цепочки действий над соответствуюшими собственными значениями исходных циркулянтных матриц. Операции сложения-вычитания рассматриваются на основе того же подхода.

Теорема. Пусть матрицы $A_{n}$ получены как результат чепочки матричных операций $\mathscr{A}$ и $f(x)$ - результат чепочки соответствующих арифметических операций над производящими функциями. Если $f_{m+l}$ отвечает матриче $T_{m+l}$, для которой проводится обращение, то потребуем, чтобъ $\left|f_{m+l}(x)\right| \geqslant c_{0}>0$ почти всюду. Тогда сингулярные числа матрии $A_{n}$ имеют асимптотическое распределение $f(x)$.

Доказательство сводится к применению лемм 10-12. 


\section{Список литературы}

1. Tyrtyshnikov E. E. Influence of matrix operations on the distribution of eigenvalues and singular values of Toeplitz matrices // Linear Algebra Appl. 1994. V. 207. P. 225-249.

2. Тыртышников E. E. Распределения и кластеры // Матричные методы и алгоритмы. М.: ИВМ РАН, 1993. С. 124-166.

3. Тыртышников E. E. Новые теоремы о распределении собственных и сингулярных чисел многоуровневых тёплицевых матриц // Докл. РАН. 1993. Т. 333. № 3. С. 300-303.

4. Замарашкин Н. Л., Тьртышников Е. Е. Распределение собственных и сингулярных чисел тёплицевых матриц при ослабленных требованиях к производящей функции // Матем. сб. 1997. Т. 188. № 8. С. 83-92.

5. Гохберг И. Ц., Крейн М. Г. Введение в теорию линейных несамосопряженных операторов. М.: Наука, 1965.

6. Гренандер У., Сегё Г. Тёплицевы формы и их приложения. М.: ИЛ, 1961.

7. Tyrtyshnikov E. E. A unifying approach to some old and new theorems on distribution and clustering // Linear Algebra Appl. 1996. V. 232. P. 1-43.

8. Avram F. On bilinear forms on Gaussian random variables and Toeplitz matrices // Probab. Theory Related Fields. 1988. V. 79. P. 37-45.

9. Parter $S$. $V$. On the distribution of the singular values of Toeplitz matrices // Linear Algebra Appl. 1986. V. 80. P. 115-130.

10. Chan $R$. Circulant preconditioners for Hermitian Toeplitz systems // SIAM J. Matrix Anal. Appl. 1989. V. 10. P. 542-550.

Институт выгислительной математики РАН

Поступила в редакцию

14.06.2001 